\title{
Impedance-based analysis of harmonic resonances in HVDC connected Offshore Wind Power Plants
}

\author{
Igor Sowa ${ }^{\mathrm{a}, \mathrm{b}}$, José Luis Domínguez-García $^{\mathrm{a}}$, Oriol Gomis-Bellmunt ${ }^{\mathrm{c}}$ \\ ${ }^{a}$ IREC Catalonia Institute for Energy Research, Jardins de les Dones de Negre 1, 2a. 08930 Sant Adrià de Besòs, Barcelona, Spain \\ ${ }^{b}$ Institute for Automation of Complex Power Systems, E.ON Energy Research Center, RWTH Aachen, Mathieustr. 10, 52074 Aachen, \\ Germany \\ ${ }^{c}$ Centre d'Innovació Tecnològica en Convertidors Estàtics i Accionaments (CITCEA-UPC), Universitat Politècnica de Catalunya UPC, \\ Av. Diagonal, 647, Pl. 2. 08028 Barcelona, Spain
}

\begin{abstract}
During the last years, the installation and planning of offshore wind farms using HVDC-links to transmit power onshore has increased. After the first HVDC connected offshore wind power plant of this type had been commissioned, the electrical harmonic resonance at the offshore AC grid was observed. The phenomenon leads to unwanted outages on both wind turbines and the HVDC transmission system. This paper aims to present the harmonic resonances in powerelectronics dominated grids such as HVDC connected wind power plants. The study focuses on harmonic frequencies identification which are excited through the resonance phenomena between the elements of the offshore AC network including power converters. The paper presents a comparison of three different methodologies existing in the literature for harmonic resonance analysis including stability analysis. Moreover, we analyse the impact of the different power converter models application. The models and methods are validated in different test cases in order to determine the relationship of such resonances with respect to the grid topology.
\end{abstract}

Keywords: wind power plant, impedance analysis, harmonic resonance, HVDC transmission, converter modelling

\section{Introduction}

Wind Power installations are increasing rapidly in the ${ }^{20}$ last years [1]. This leads wind power to become the most ${ }_{28}^{27}$ relevant technology among non-conventional renewable energy sources [2]. Due to space availability and better wind ${ }^{29}$ speed conditions, wind farm trend is to locate them offshore. For taking advantage of all sea space and better wind conditions, wind turbines are being installed at large ${ }_{33}$ distances from shore, with a clear trend to increase [2]. For ${ }^{33}$ long distances and large cable power transmission needs, ${ }_{35}^{34}$ HVDC technologies are more cost-effective than conventional AC system, for power transmission [6]. HVDC technologies increase controllability of the system and remove ${ }_{38}$ the reactive power compensation requirements, which are ${ }_{39}$ critical for offshore and remote locations.

HVDC connection decouples the offshore AC network ${ }_{41}^{40}$ (offshore wind farm) from the main AC grid. Thus, the ${ }_{42}$ offshore wind farm dynamics are mainly dominated by the ${ }_{43}$ cables, power converters, filters and power transformers. ${ }_{44}$ Such dynamics do not have any support provision from $a_{45}^{44}$ large AC grid, potentially leading to unexpected responses. The cables and power converters dynamics may engage ${ }_{47}^{46}$ causing harmonic resonances, oscillations and, even, instabilities in the offshore AC network.

Email address: jldominguez@irec.cat Corresponding author 51 (José Luis Domínguez-García)
Concerns for harmonics rises from power quality requirements which are introduced to prevent from negative effects on electrical equipment which are sensitive to poor power quality. Poor power quality leads to damages of equipment, life-time reduction and other dynamics [4]. Harmonics in power systems are produced due to many phenomena, for example, ferroresonance, magnetic saturation, sub-synchronous resonance, and nonlinear and electrically switched loads [7]. This paper focuses on the impact of power converters existing in an offshore wind power plants, as the ones used in the wind turbines and in the HVDC transmission system on the harmonic content of the offshore grid and other potential interactions as harmonic resonances. Harmonic resonant conditions may occur when the harmonic wave contains frequencies similar to the natural ones of the electrical network, which are mainly dominated by the inductances and the capacitances of the grid [5]. When such frequency is close, the grid magnifies such response due to amplitude matching and fault excitation [9].

The abnormal and unexpected behavior was observed in the operation of the first high-power HVDC connected offshore wind power plant (WPP), where the presence of harmonic resonances under normal operation lead to abnormal responses of the system as failures, instabilities, shut-downs or even damage of components [3]. The problems found were not considered during planning period 
of that WPP. Thus, due to very strong development of $f_{104}$ HVDC connected offshore wind farms, new methods of in-105 vestigation and analysis for resonance should be developed ${ }_{106}$ or current ones should be extended.

This paper aims to analyse different existing measures108 in impedance-based harmonic resonance analysis, crucial ${ }_{109}$ in the systems subjected to resonance occurrences such ${ }_{110}$ as offshore WPP connected by HVDC link. The two main 11 categories of measures in the analysis are (i) methodologies of impedance-based resonance analysis, and (ii) techniques of converter modelling. The comparison and impact of those measures on the overall harmonic resonance analysis ${ }^{112}$ are studied.

The impact of different network topologies on the ${ }^{114}$ methodology and model measures is considered. Finally, ${ }^{115}$ the Nyquist stability analysis with phase margin crite- ${ }^{116}$ rion verifies the methods and models consistency. The ${ }^{117}$ Nyquist analysis couples the impedance based studies with ${ }^{118}$ stability assessment method within the assumed measures. ${ }_{120}^{119}$

\section{Methods for harmonic resonance analysis}

In this section, the main methods (or techniques) ap- ${ }^{122}$ plied for harmonic resonance analysis are introduced and ${ }^{123}$ described.

\subsection{Frequency Sweep}

Frequency Sweep (or Frequency Scan) analysis is a characterization of the system equivalent impedance at $a_{126}$ bus in the system as a function of frequency [9][16]. This ${ }_{127}$ method analyse the equivalent impedance seen at a cer-128 tain bus for a wide range of frequencies. As the result, a curve of impedance of the whole system in frequency do-130 main is obtained. The peaks in the curve suggest frequen-131 cies when parallel resonance occurs (very high impedance ${ }_{132}$ at certain frequency) while dips indicate the frequencies ${ }_{13}$ when series resonance occurs (very low impedance at cer-134 tain frequency).

In Wind Power Plants frequency scans are often done 136 at various grid locations or at the collector bus [9]. Sin-137 gle value of identified peak impedance does not deter-138 mine if a dangerous harmonic resonance occurs. For har-139 monic problems, there must also be a sufficient level of ${ }_{140}$ harmonic source voltages or currents at or near the res-141 onant frequency to excite harmonic resonance [9]. Also,142 the impedance value itself has to be analysed in particular ${ }_{143}$ case to identify a degree that could cause a harm.

\subsection{Harmonic resonance Modal Analysis (HRMA)}

The method applies modal analysis on the admittance ${ }^{147}$ matrix - $\boldsymbol{Y}$. Resonances are identified through the ad-148 mittance matrix calculated for particular frequency. In principle, when the admittance matrix tends to singularity, it means that the system might experience parallel ${ }_{149}$ resonance. Such singularity may be computed through ${ }_{150}$ linear algebra methods, as the matrix $\boldsymbol{Y}$ becomes singular when at least one of the eigenvalue is zero. The eigenvalues obtained from the modal analysis correspond to certain mode of harmonic resonance, therefore the methods allows to identify critical resonance modes.

The admittance matrix on the network is constructed for certain frequency $\boldsymbol{Y}_{f}$. Admittance matrix fulfils equation:

$$
V_{f}=Y_{f}^{-1} I_{f}
$$

where $\boldsymbol{Y}_{\boldsymbol{f}}$ is the network admittance matrix, $\boldsymbol{V}_{\boldsymbol{f}}$ is the nodal voltage and $\boldsymbol{I}_{\boldsymbol{f}}$ is the nodal current injection. The indexes $f$ refers to the frequency.

To investigate if $\boldsymbol{Y}_{\boldsymbol{f}}$ approaches singularity, the theory of eigen-analysis is applied. The exemplary admittance matrix of one of the studied sytem is included in the AppendixC.

According to [15], matrix $\boldsymbol{Y}_{\boldsymbol{f}}$ can be decomposed into (index $f$ is neglected in the next equations for simplicity):

$$
Y=L \Lambda T
$$

where $\boldsymbol{\Lambda}$ is the diagonal eigenvalue matrix and $\boldsymbol{L}$ and $\boldsymbol{T}$ are the left and right eigenvector matrices.

Defining $\boldsymbol{U}=\boldsymbol{T} \boldsymbol{V}$ as the modal voltage vector and $\boldsymbol{J}=\boldsymbol{T} \boldsymbol{I}$ as the modal current vector, the equation can be derived:

$$
\boldsymbol{U}=\boldsymbol{\Lambda}^{-1} \boldsymbol{J}
$$

where $\Lambda^{-1}$ has the unit of impedance and is named modal impedance $Z_{m}$. From Equation 2, one can easily identify the location of resonance in the modal domain due to corresponding modal currents and voltage. If $\lambda_{1}=0$ or is very small, a small injection of modal 1 current $J_{1}$ will lead to a large modal 1 voltage $U_{1}$ [10]. Thus, we can identify that a resonance takes place for specific mode (or modes) and it is not related to a particular bus injection since the values are in modal domain. The smallest eigenvalue is called the critical mode of harmonic resonance and its left and right eigenvectors are the critical eigenvectors.

The modal currents $J$ are a linear projections of the physical currents in the direction of the first eigenvectors by $\boldsymbol{J}=\boldsymbol{T} \boldsymbol{I}$. Also the physical nodal voltages are related to the modal voltages by $\boldsymbol{V}=\boldsymbol{L} \boldsymbol{U}$. More details in [10]. In summary, the critical eigenvectors characterize the excitability of the critical mode (right critical eigenvector) and observability of the critical mode (left critical eigenvector). The excitability and observability of modes are characterized with respect to the location.

It is also possible to combine the excitability and observability into a single index according to the theory of selective modal analysis [8]:

$$
V=L \Lambda^{-1} T I
$$

However, this approximation is made possible because $1 / \lambda_{1}$, the critical modal impedance, is much larger than 
the other modal impedances. If there are more impedances 193 at the similar level as critical impedance, we will observe ${ }_{194}$ some inaccuracies in the results.

Assuming one critical modal impedance, much larger than the others, the diagonal elements of the above matrix $\boldsymbol{L T}$ characterize the combined excitability and observability of the critical mode at the same bus. They are called participation factors (PF's) of the bus and are defined as ${ }_{196}^{195}$ follows [10]:

$$
P F_{b m}=L_{b m} T_{m b}
$$

where $b$ is the bus number and $m$ is the mode number. Thus, we can observe which components are more involved in a resonance than other. From these results we can conclude where a resonance can be observed more easily or how far the resonance can propagate in the system [10].

To summarize, from the calculation on the basis of admittance matrix (decomposition into eigenvectors and eigenvalues) and the approximation above (Equations 2197 - 5) we obtain: the set of participating factors for each ${ }_{198}$ bus for each critical mode (the modes when the modal ${ }_{199}$ impedance peaks), which occurs for certain frequency at 200 certain number of mode.

\subsection{Impedance-based stability evaluation method}

This stability criterion for harmonic resonance stability 204 based on Nyquist stability criterion is described in [12] and ${ }^{205}$ is theoretically well-established and investigated for real ${ }^{206}$ applications [3]. For the implementation only frequency ${ }^{207}$ dependent impedances of converter are needed, including ${ }^{208}$ passive elements impedance and impedance changes due ${ }^{209}$ to active controls [3]. The method avoids the need to re-210 model each inverter and repeat its loop stability analysis ${ }^{211}$ when the grid impedance changes [12]. This method is ${ }^{212}$ considered as very fast and can evaluate new topology if ${ }^{213}$ any switching action occurs [3]. The simplicity is achieved ${ }^{214}$ by aggregation of all wind farms with their controllers into ${ }^{215}$ one element. Then, the aggregated system is evaluated ${ }^{216}$ by Nyquist stability criterion interpreted in Bode diagram ${ }^{217}$ that provides information about frequency and phase mar- ${ }^{218}$ gin.

With the proper data and assumptions described ${ }^{220}$ above, we use the simple model to evaluate the stability ${ }^{221}$ consisting of voltage source with internal impedance and ${ }^{222}$ the impedance of the grid (Figure 1) [3, 12].

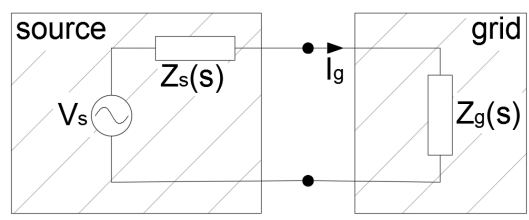

Figure 1: Model for stability analysis consisting of voltage source 230 with internal impedance (the source) and grid impedance (the grid) ${ }_{231}$
In such a network, the current $I_{g}$ depends on both $Z_{s}$ and $Z_{g}$ impedances:

$$
I_{g}=\frac{V_{s}(s)}{Z_{s}(s)+Z_{g}(s)}=\frac{V_{s}(s)}{Z_{g}(s)} \frac{1}{1+\frac{Z_{s}(s)}{Z_{g}(s)}}
$$

The equation of the network $I_{g}$ current (Eq. 6) can be expressed as loop gain for the system in the Figure 2.

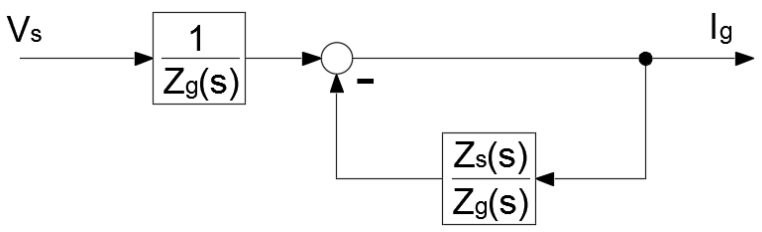

Figure 2: Loop gain corresponding to the stability model in the Figure 1.

On the basis of the Eq. (6), we conclude that the system is stable if the source has zero and the load infinite output impedance. For stability, the value of ratio $\left|Z_{s}(s) / Z_{g}(s)\right|$ has to be at least below 1 to for all frequencies [12] in other words the system is stable if $Z_{s}(s) / Z_{g}(s)$ satisfies the Nyquist stability criterion [13].

The first problem with the model above is the division point between the source $\left(Z_{s}\right)$ and the grid $\left(Z_{g}\right)$. The best point of division is still under investigation [3]. A change of this division brings changes to both aggregate impedances, therefore could significantly influences the results. In this study the network is divided behind the HV transformer from the HVDC link point of view (Bus2) (see Figure 6).

There is also other conceptual problem with the presented approach. As either the WT converter or HVDC converter could be treated as the source, the results about stability could be very different [12]. In this study we perform only one approach where the aggregated WT converter is treated as the source and HVDC link converter as the grid and the point of division is defined always as described above. The WT has been chosen as source because most of the operation time the wind turbine deliver power to the wind farm collection grid and the HVDC converter absorbs it to deliver to main AC grid.

Finally, the stability criterion requires frequency impedances of converters which could be modelled as either voltage or current sources. The problems and details about these two models are explained in [12]. The source part and the grid part of the network can be modelled by its Thevenin equivalent circuit (voltage source) or Norton equivalent circuit (current source). However, in frequency domain, Norton and Thevenin models are equivalent.

Stability assessment. As stated in the previous sections the stability assessment comes down to the evaluation of Nyquist stability criterion of the impedances ratio. In our study, the results of those impedances will be analysed in the Bode diagrams due to the ease of resonance frequency 
identification. Bode diagrams combine all necessary data287 including information about frequency which is missing in ${ }_{288}$ the Nyquist plots.

The evaluation of the Nyquist stability criterion in 290 the Bode diagram depends on two crucial points of the 291 Bode curves: the zero- $\mathrm{dB}$ crossing point and $-180^{\circ}$ cross-292 ing point.

Zero- $\mathrm{dB}$ crossing point is the point when the Bode mag-294 nitude curve crosses the $0 \mathrm{~dB}$ value. In our case, we evalu-295 ate the ratio of the grid and the source impedances. Since 290 the ratio of two values in logarithmic scale $(\mathrm{dB})$ is subtrac-297 tion in the linear scale, the zero-dB crossing occurs when 298 the values are the same (subtraction of two equal numbers299 gives zero). Therefore, in our case, the zero- $\mathrm{dB}$ crossing 300 points are when the impedances are the same i.e. at the in-301 tersection points of the source impedance magnitude curve 302 and the grid impedance magnitude curve.

The second crucial point for stability evaluation is the $3_{30}$ $-180^{\circ}$ crossing point which is the point when the Bode an-305 gle curve crosses $-180^{\circ}$. Once again, due to the same reason 306 as for zero-dB crossing, the curve that crosses the level of $-180^{\circ}$ is the result of the grid and the source impedance angles subtraction. To evaluate this condition, the concept of phase margin is introduced. It is well-known idea of the Nyquist stability criterion that offers the possibility 307 of more practical quality assessment of system stability. $\mathrm{In}_{308}$ our case, the phase margin will be calculated according to $_{309}$ following equation [3]:

$$
\phi_{m}=180^{\circ}-\Delta \phi
$$

where $\Delta \phi$ is the phase difference between considered ${ }^{313}$ curves in degrees. According to control theory, the larger ${ }^{314}$ the distance of the locus from the critical point, the far-315 ther is the closed loop system from the stability. As the ${ }^{316}$ measure of this distance, exactly the phase margin is eval-317 uated.

The model in the study is based on linearisations and ${ }^{319}$ other assumptions introducing uncertainties (such as im-320 perfect models of electrical components), therefore the calculated angle difference do not reach the theoretical value ${ }^{321}$ of $180^{\circ}$ (and the phase margin does not reach $0^{\circ}$ ). Accord- ${ }^{322}$ ing to [3], that gives some insight of industrial experience ${ }^{323}$ with the evaluation of the phase margin, the value of $30^{\circ} 324$ as safety margin is introduced. If the phase margin calculated in such a way is below safety margin, the system is assumed to be possibly unstable.

To sum up, as the result of stability assessment, we obtain, for each intersection, phase margin marking either stable or unstable operation. As aforementioned, the stability assessment is performed for specified point of division and specified the source and the grid sides.

\section{Modelling of elements}

\subsection{Transformers, cables, filter reactors} grid models for very high frequencies are generally not nec- 329 essary. In this study, two- and three- winding transformers impedances will be represented simply by its inductance as $Z_{t r}\left(j \omega_{f}\right)=j X_{t r}\left(\omega_{f} / \omega_{1}\right)$, where $X_{t} r$ corresponds to fundamental frequency reactance. The skin effect and eddy currents affect the resistance at higher frequencies, therefore we do not consider these effects.

Filter reactors modelling is important since it significantly affects the tuning of whole system. In the models presented, series resistance of LCL filters are neglected (equals zero), therefore they are modelled as series inductances.

Modelling of cables is important in harmonic analysis since they are very significant source of capacitance in considered grids. For harmonic frequencies up to $3000 \mathrm{~Hz}$ the resistance of cables will increase, meanwhile the variation of inductances and capacitances may be ignored. Cables may be represented as PI sections (one single or multiple in series) and distributed parameters. In this study, a single PI model per cable is considered, which may be described by:

$$
\begin{array}{r}
Z_{\text {cable }}\left(j \omega_{f}\right)=R_{\text {cable }}+j\left(\omega_{f} / \omega_{1}\right) L_{\text {cable }} \\
Y_{\text {cable }}\left(j \omega_{f}\right)=j\left(\omega_{f} / \omega_{1}\right) C_{\text {cable }}
\end{array}
$$

\subsection{Power converters}

Modelling of power converters is the most crucial and challenging within all elements. Power converters devices are very nonlinear and their impedance behaviour depends on many factors. The exact model should be derived on the basis of control codes and ideally also on the basis of measurements performed on the real device.

Control codes are very unique and never published by the manufacturers. They are considered as an intellectual property and thus the determination of the exact impedances is very difficult [3]. There are also more simple approaches to face the problem of converter modelling. The principles presented below are considered for frequency domain analysis.

\subsubsection{Voltage Source (VS) and Current Source (CS) mod- els}

It is common to approach modelling of converters as either current or voltage source (Figure 3).
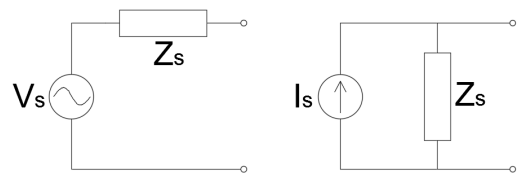

Figure 3: Ideal voltage source and current source models for converters modelling.

There is very important fact to be considered for both approaches in frequency domain analysis. According to circuit theory, ideal voltage source internal resistance equals zero (short-circuit). On the other hand, the ideal current source internal resistance is infinite (open-circuit). 
In this study, models with either ideal current sources360 or voltage sources are considered for comparison in $\mathrm{FS}_{367}$ method and HRMA method. For those models ("VS" or 368 "CS"), internal impedance of source $Z_{s}$ is zero or infinite, respectively.

\subsubsection{Frequency dependent impedance model $Z(s)$}

The models of either ideal voltage/current sources are ${ }_{330}^{369}$ very important; however, for the resonance analysis the ${ }_{371}$ value of series impedance (in case of voltage source) or $_{372}$ parallel impedance (in case of current source) is crucial $_{373}$ The third converter model utilized is the approach devel- ${ }_{374}$ oped in [11] and [14] of frequency dependent impedance of f $_{375}^{374}$ converters.

The authors, by applying appropriate modelling meth- ${ }^{376}$ ods, such as harmonic linearisation presented in [17, 18], obtain impedance models valid below and above the fundamental frequency [11]. Each converter is described by positive- and negative-sequence impedances without cross coupling [19]. The Park's transformation is crucial to derive the converters impedances equations.

The assumed converters modelled are [11]: 2-level VSC Wind Turbine DC/AC inverter and the same type of $_{378}$ HVDC AC/DC rectifier. Models of these converters are 379 then used in the simulations.

Wind turbine converter (inverter). For the control purposes, wind turbine converter is controlled as current source. Due to this fact, the device behaves more like cur- ${ }^{380}$ rent source and the control will be modelled in this way. Reactive power supply and voltage regulation of the model is not considered. A phase-locked loop (PLL) is included ${ }_{383}^{382}$ in the model for AC bus synchronisation [11].

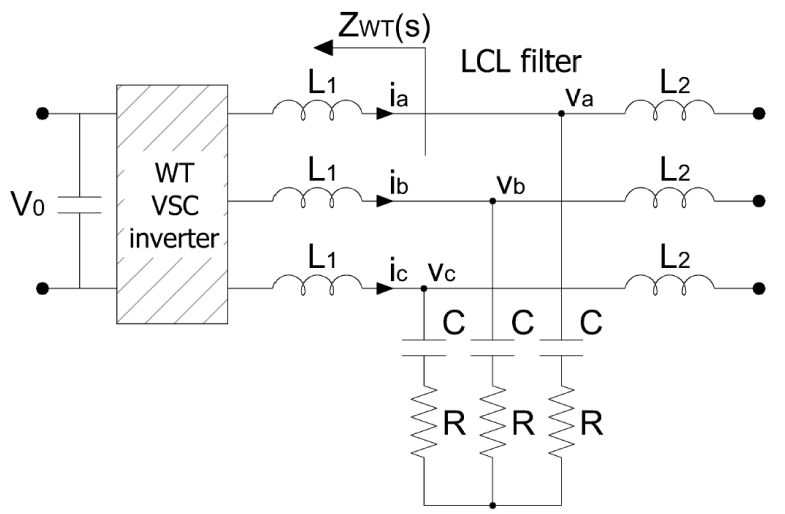

Figure 4: Simplified diagram of aggregated wind turbine converter (inverter) with LCL filter.

The wind turbine model is described in $d q$-frame. As mentioned, the current control scheme is used. The reference value is the value of current provided be the $\mathrm{DC}_{387}^{386}$ link voltage regulator. The current compensator transfer function is given:

$$
H_{i}(s)=K_{p}+\frac{K_{i}}{s}
$$

The PLL is implemented using PI regulator. Including the integrator to convert frequency into angle, the PLL compensation transfer function becomes:

$$
H_{p}(s)=\left(K_{p}+\frac{K_{i}}{s}\right) \frac{1}{s}
$$

The values of parameters are specified in Section 4 . For the stability study, the wind turbines are lumped into one device (one impedance). The output impedance of WT inverter is developed using the harmonic linearization method described in [18]. As the result, converters are represented by positive-sequence and negative-sequence impedances without cross coupling [19]. Providing constant DC bus voltage (as the reference) the impedances become [11]:

$$
\begin{aligned}
& Z_{p}(s)=\frac{H_{i}\left(s-j \omega_{1}\right) V_{0}+\left(s-j \omega_{1}\right) L_{1}}{1-T_{p l l}\left(s-j \omega_{1}\right)\left[1+H_{i}\left(s-j \omega_{1}\right) I_{1} V_{0} / V_{1}\right]} \\
& Z_{n}(s)=\frac{H_{i}\left(s+j \omega_{1}\right) V_{0}+\left(s+j \omega_{1}\right) L_{1}}{1-T_{p l l}\left(s+j \omega_{1}\right)\left[1+H_{i}\left(s+j \omega_{1}\right) I_{1} V_{0} / V_{1}\right]}
\end{aligned}
$$

where $\omega_{1}$ is fundamental angular frequency, $T_{p l l}(s)$ is the loop gain of $d q$-frame PLL defined by:

$$
T_{p l l}(s)=\frac{V_{1} H_{p}(s)}{2\left[1+V_{1} H_{p}(s)\right]}
$$

and $H_{i}$ and $H_{p}$ are the current an PLL compensator transfer functions, as defined before.

HVDC link converter (rectifier). In case of HVDC converter, the device is controlled to behave as a voltage source at the ac terminals [20]. Figure 5 demonstrate the model for HVDC converter impedance calculation.

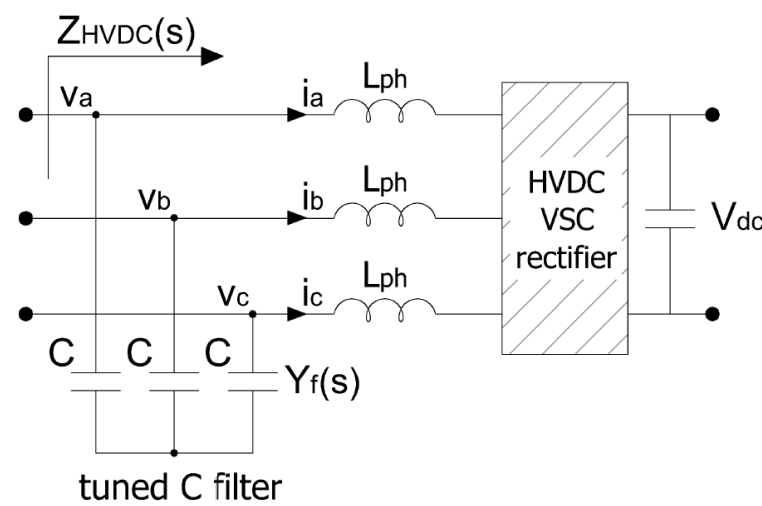

Figure 5: Simplified diagram of HVDC-link VSC converter (rectifier) with tuned $\mathrm{C}$ filter and phase reactor.

The HVDC rectifier voltage control is performed by a PI regulator in the dq-reference frame:

$$
H_{v}(s)=K_{p}+\frac{K_{i}}{s}
$$


The current loop is embedded within the voltage loop and the current compensator transfer function is defined as:

$$
H_{i}(s)=K_{p}+\frac{K_{i}}{s}
$$

Other control approaches could be incorporated but are not considered. The values of parameters are included in Section 4.

Again, the assumption of constant DC-link voltage $\left(V_{d} c\right)$ is made. The resulting positive- and negativesequence input impedance are given by [11]:

$$
\begin{aligned}
Z_{p}(s) & =\frac{H_{i}\left(s-j \omega_{1}\right) V_{d c}+s L_{p h}}{1+Y(s)\left[H_{i}\left(s-j \omega_{1}\right) V_{d c}+s L_{p h}\right]+T_{p}(s)} \\
Z_{n}(s) & =\frac{H_{i}\left(s+j \omega_{1}\right) V_{d c}+s L_{p h}}{1+Y(s)\left[H_{i}\left(s+j \omega_{1}\right) V_{d c}+s L_{p h}\right]+T_{n}(s)}
\end{aligned}
$$

where $\omega_{1}$ is fundamental angular frequency, $Y(s)$ is admittance of the ac filter, in our case equals $Y(s)=s C$. $T_{p}(s)$ and $T_{n}(s)$ are defined as:

$$
\begin{aligned}
& T_{p}(s)=\left[H_{i}\left(s-j \omega_{1}\right)+j K_{i d}\right] H_{v}\left(s-j \omega_{1}\right) V_{d c} \\
& T_{n}(s)=\left[H_{i}\left(s+j \omega_{1}\right)-j K_{i d}\right] H_{v}\left(s+j \omega_{1}\right) V_{d c}
\end{aligned}
$$

and $H_{i}(s)$ and $H_{v}(s)$ are the current and voltage compensator transfer functions defined before.

\section{Results}

\subsection{System description}

In the simulations for harmonic resonance study we consider offshore wind power plant with VSC-HVDC connection to the onshore grid. The layout of the $400 \mathrm{MW}$ WPP is presented in the Figure 6.

Each of four branch is formed by ten 10-MW wind ${ }^{427}$ turbines with a terminal voltage of $690 \mathrm{~V}$. The aggregated ${ }^{428}$ model of each branch is used where each set of ten tur- ${ }^{429}$ bines is lumped and modelled as a single aggregated tur- ${ }^{-430}$ bine, represented by a $100 \mathrm{MW}$ turbine. Each aggregated ${ }^{431}$ turbine is connected to the LCL filter and further to the ${ }_{432}$ elements of collection grid: $690 \mathrm{~V} / 33 \mathrm{kV}$ transformer, $8 \mathrm{~km}_{433}$ collector cable $(33 \mathrm{kV}), 150 \mathrm{kV} / 33 \mathrm{kV} / 33 \mathrm{kV}$ three winding ${ }_{434}$ transformer with YN-dd configuration, $150 \mathrm{kV}$ transmis- ${ }_{435}$ sion cable with a length of $58 \mathrm{~km}$. The $150 \mathrm{kV}$ transmission cable is tied to the VSC-HVDC rectifier through ${ }^{4}{ }^{436}$ $150 \mathrm{kV} / 150 \mathrm{kV}$ transformer and a phase reactor with an ${ }^{437}$ tuned shunt capacitor filter.

All of the parameters are converted to the $150 \mathrm{kV}$ equivalent voltage level (see AppendixA). The impedances of ${ }^{439}$ VSC-WT inverters and VSC-HVDC rectifier are calcu- ${ }^{40}$ lated on the basis of three different methods presented in $^{441}$ Section 3.2 .

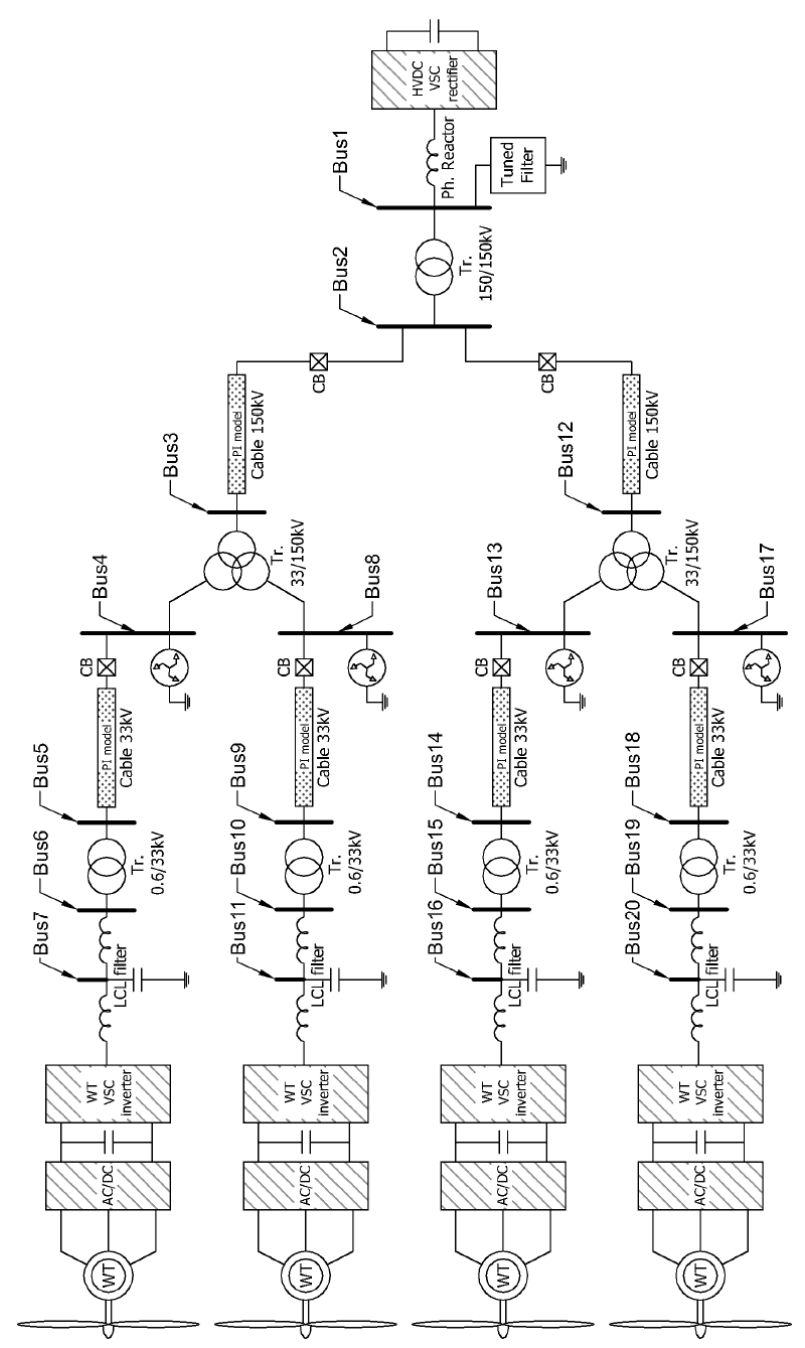

Figure 6: Wind Power Plant of $400 \mathrm{MW}$ considered in the study.

Topology cases. In the study, we approach comparison between different topologies. There are three topology cases examined. The difference between three topology depends on the number of included branches with aggregated wind turbines (1, 2 or 4 branches). The buses in the figures have assigned numbers which we employ in further analysis.

- Case 1 model consist of one aggregated WT. The other three branches are disconnected by circuit breakers at the lower side of the three-winding transformers.

- Case 2 includes one more aggregated WT than Case 1. The second WT branch is connected to the first three-winding transformer.

- Case 3 consists of all elements in the networks. All branches are activated, therefore all elements are included in analysis.

Power converters models. In the simulations, we refer the following names to the different models of converters as 
follows: $V S$ the model where both WT and HVDC con-463 verters are modelled as voltage sources (Section 3.2.1),464 $C S-W T$ or $C S$ where WT converter is modelled as a current source and HVDC converter is still represented by 465 VS, $Z(s)$ where both converters are represented by non-466 linear impedance models (Section 3.2.2). The parameters ${ }_{467}$ used in such models can be found in AppendixB.

The results of impedance for both Z(s) model convert- ${ }_{469}^{468}$ ers are demonstrated in the Figure 7 for WT-converter and ${ }_{470}$ in the Figure 8 for HVDC converter. Both plots include ${ }_{471}$ curves of impedance magnitude and impedance angle for ${ }_{472}$ positive-sequence and negative-sequence in the domain of ${ }_{473}$ frequency.
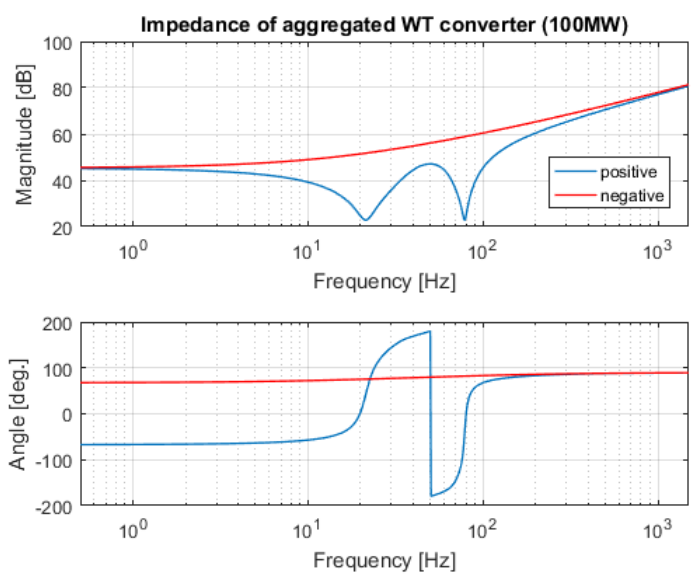

Figure 7: Results of the WT converter nonlinear impedances in frequency domain (positive- and negative-sequences of impedance).
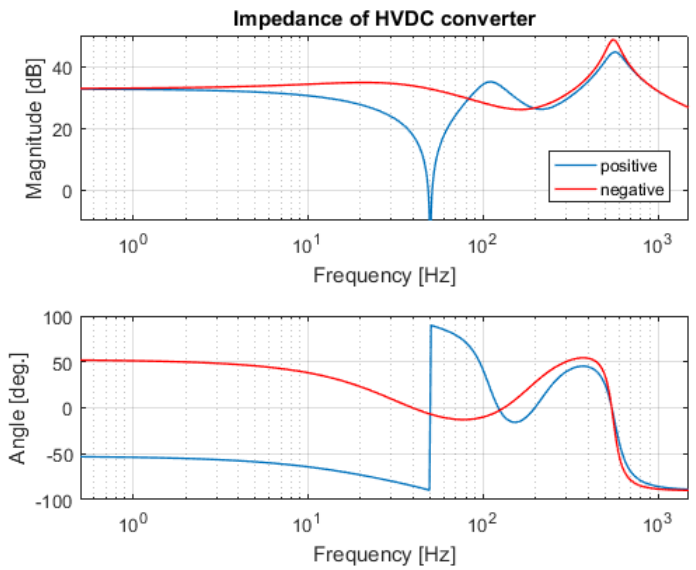

Figure 8: Results of the HVDC-link converter nonlinear impedances in frequency domain (positive- and negative-sequences of impedance).

For both converters, we note sharp changes in the val-

and negative-sequences are very close to each other for all four plots.

\subsection{Comparison of resonance frequencies between differ- ent topology cases and converter models}

First, the comparison of resonance frequencies for three topology cases and three converter models is performed. The comparison is performed based on two methods: Frequency Sweep (Section 2.1) and Harmonic Resonance Modal Analysis (Section 2.2). Secondly, on the basis of HRMA we spot the buses that have the most significant influence on the particular resonance frequencies. Due to the paper length limitations, the Case 1 and Case 2 are excluded. The excluded results are presented in [21].

\subsubsection{Case 3}

Frequency Sweep. Figure 9 presents the frequency sweep curves. All three models are included. The bus of observation is Bus7 - in other words, the impedance is seen from that point of the network.

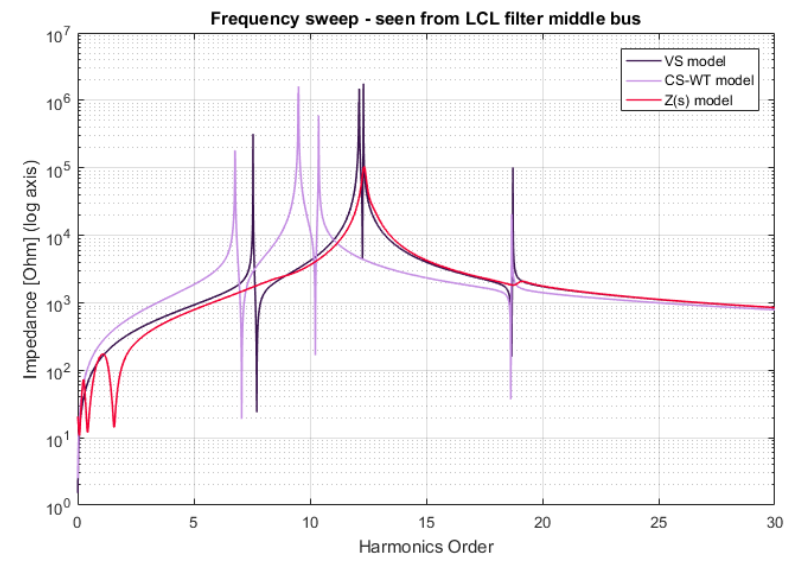

Figure 9: Case 3: Frequency sweep curves (2) for three models (seen from bus 7 ).

\begin{tabular}{|c|c|c|}
\hline Conv Model & Freq order & Peak Imp $(\mathrm{k} \Omega)$ \\
\hline \multirow{5}{*}{ VS } & 7.55 & 318 \\
\hline & 12.1 & 941 \\
\hline & 12.12 & 1491 \\
\hline & 12.3 & 1769 \\
\hline & 18.73 & 102 \\
\hline \multirow{5}{*}{ CS-WT } & 6.78 & 180 \\
\hline & 9.49 & 1288 \\
\hline & 9.51 & 1606 \\
\hline & 10.37 & 595 \\
\hline & 18.68 & 21 \\
\hline \multirow{2}{*}{$\mathrm{Z}(\mathrm{s})$} & 12.33 & 104 \\
\hline & 19.13 & 2 \\
\hline
\end{tabular}


Harmonic Resonance Modal Analysis. Following Figure 10 shows the HRMA results of modal impedance curves for all modes separately. The graphs of maximum modal impedances for each frequency in the scope and critical modes curves alone are excluded from this paper and can be found in [21]. Critical modes are the ones that determine the resonances and we can notice them from the Figure 10 (modes: 8, 12, 16, 19 - the same for all three models).
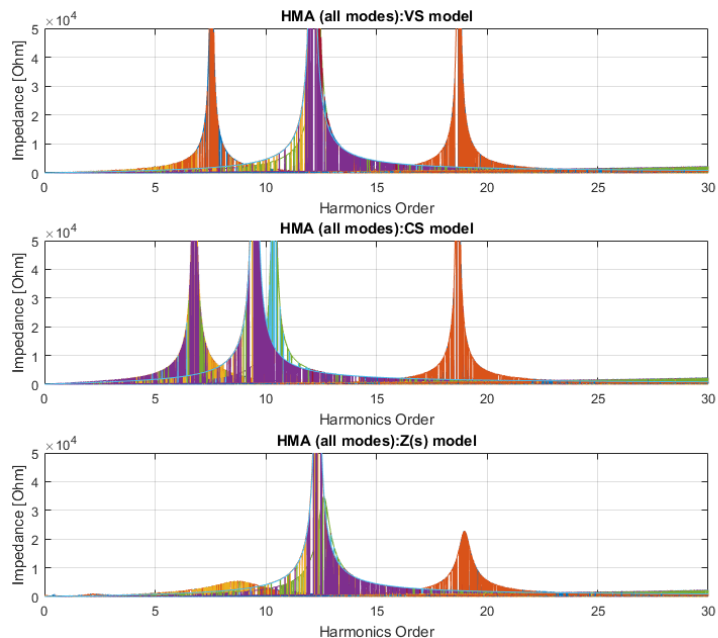

Figure 10: HRMA method all modes impedance curves for three models (Case 3).

The values of resonant frequencies and their critical ${ }_{503}^{502}$ impedances for Case 3 are presented for each resonance frequency in Table 2. The Figure 11 shows distribution of ${ }_{504}$ participation factors within the buses.

Table 2: HRMA numerical results of resonant frequencies and corre- ${ }^{506}$ sponding peak impedances for three models (Case 3).

\begin{tabular}{ccccc} 
& Order & Mode & Imp. $(\mathbf{k} \Omega)$ & Ang. (deg) \\
\hline \hline \multirow{4}{*}{ VS } & 7.55 & 8 & 6050 & -83.4 \\
& 12.1 & 16 & 2484 & -80.8 \\
& 12.12 & 19 & 2753 & 82.4 \\
& 12.3 & 12 & 7050 & 64.5 \\
CS-WT & 18.73 & 8 & 5997 & 57.5 \\
\hline \multirow{6}{*}{ Z $(\mathbf{s})$} & 6.78 & 10 & 1511 & 85.4 \\
& 9.49 & 16 & 4054 & -66.1 \\
& 10.51 & 20 & 3008 & 76.8 \\
& 18.68 & 12 & 2567 & -77.3 \\
& 12.34 & 19 & 2114 & -76.7 \\
\hline
\end{tabular}

\subsection{Different topology cases for particular models}

In this section we compare the results of different topol- ${ }_{508}$ ogy cases for particular models. Each model is considered ${ }_{509}$ separately. In the paper we present only the results of $\mathrm{VS}_{510}$ model due to the paper length limitations. The other $\mathrm{two}_{511}$
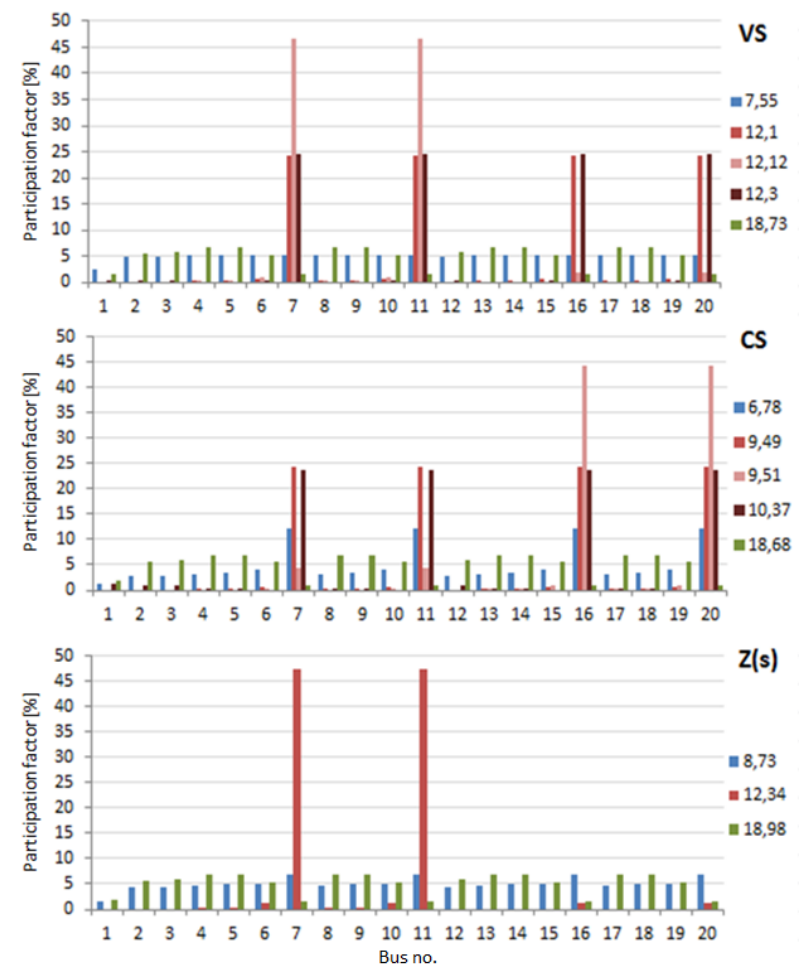

Figure 11: HRMA participation factors distribution for three models (Case 3).

models (CS-WT and Z(s)) are included in [21]. The aim of this section is to identify patterns and any similarities between the topology cases within each model.

\subsubsection{VS model}

FS and HRMA. Figure 12 presents the frequency sweep curves for all three topology cases. We sort the frequencies in the three groups like indicated in the figure.

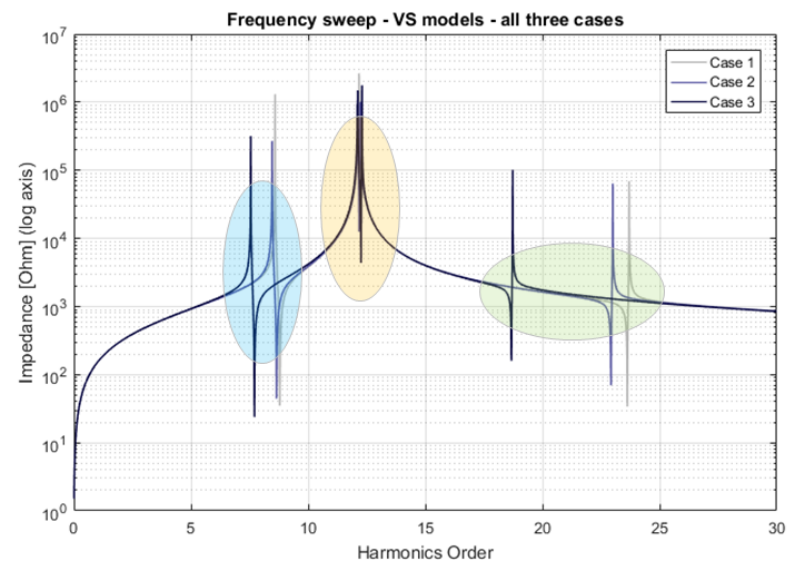

Figure 12: Frequency sweep curves for VS model in three topology cases (seen from bus 7 ).

The frequencies calculated in HRMA are the same to those obtained from frequency sweep, therefore the graph is excluded. However, we also have at our disposal the values of participation factors indicating the excitability 
and observability of the buses in the network. The values529 of PFs for three topology cases for VS mode are presented ${ }_{530}$ as bar graph in the Figure 13. tribute the most to the particular resonances at each topot-ss ogy case.
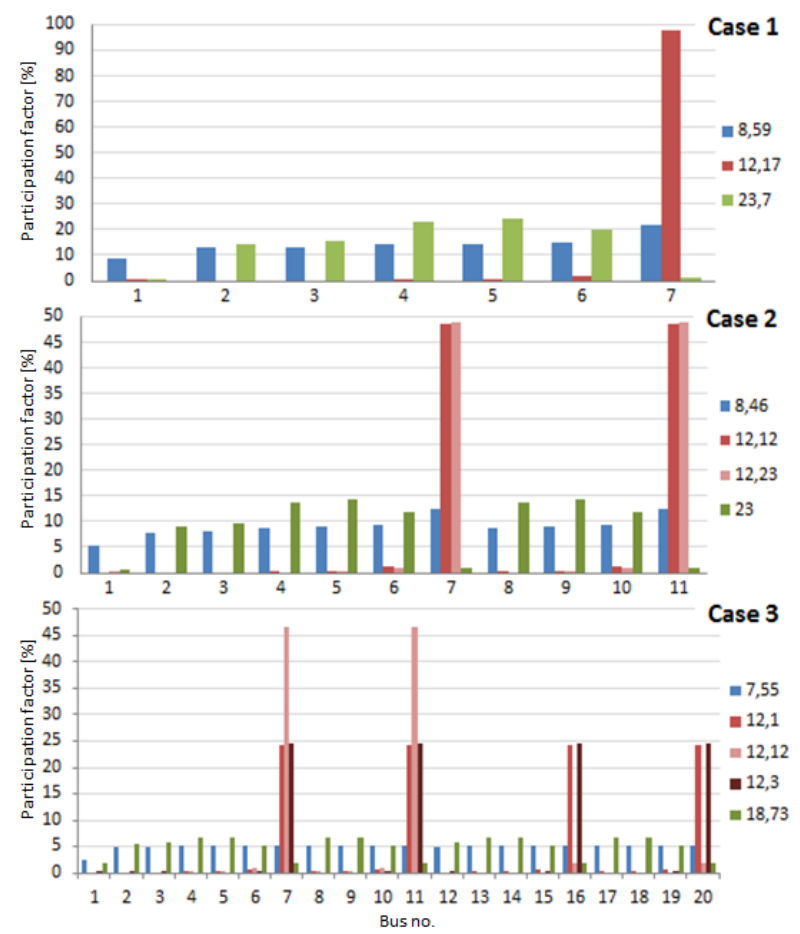

Figure 13: HRMA PF's distribution for VS model in topology cases.

Table 3: FS and HRMA numerical results of resonant frequencies for VS model in three topology cases with the dominant bus(es) assigned.

\section{Frequency order}

\begin{tabular}{cccc} 
Case 1 & Case 2 & Case 3 & Middle LCL Buses $(7)$ \\
\hline \hline 8.59 & 8.46 & 7.55 & \\
12.17 & 12.12 & 12.1 & Middle LCL Buses $(7)$ \\
& 12.23 & 12.12 & \\
& & 12.3 & \\
23.7 & 23.0 & 18.73 & cable $33 \mathrm{kV}$ terminal buses $(4,5)$ \\
\hline
\end{tabular}

\subsection{Stability study of $Z(s)$ model with respect to topology cases}

This section presents the results of stability analysis for the three different topology cases. The principles of stability analysis are described in Section 2.3. Only the last model of the network is utilized i.e. the model containing the nonlinear impedances of the converters $\mathrm{Z}(\mathrm{s})$ model. This model is considered as more accurate than two oth- ${ }^{-535}$ ers (VS and CS-WT) presented in the paper. In short, the ${ }^{536}$ stability is evaluated on the basis of phase margin plotted in the Bode diagrams.

\subsubsection{Stability analysis of Case 3}

The system impedance dynamics for source and grid is plotted on the Bode diagrams (Figures 14 and 15). Intersections of both sequences for grid and source are marked. The point of division is, as described, behind the HV transformer, looking from HVDC-link side.
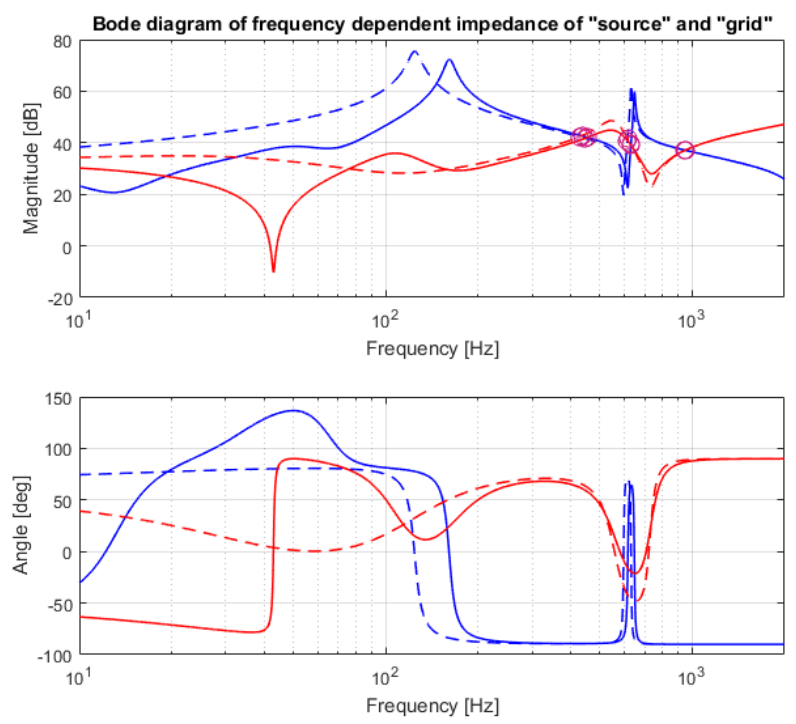

Figure 14: Case 3: Bode diagram of frequency dependent positiveand negative-impedances of grid and source
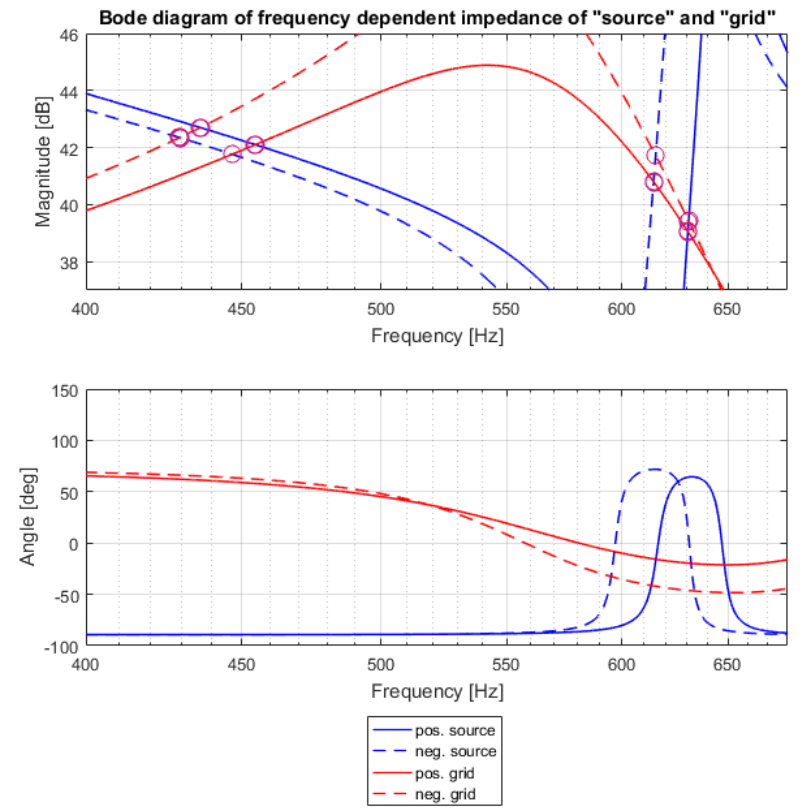

Figure 15: Case 3: Bode diagram of frequency dependent positiveand negative-impedances of grid and source- zoomed area.

The stability assessments by phase margin are gathered for all intersections (resonant frequencies) in the Table 4. 
Table 4: Case 3: Resonance frequencies and the phase margins assigned.

\begin{tabular}{cc} 
Frequency order & Phase Margin (deg) \\
\hline \hline Positive Grid - Positive Source \\
9.09 & 32.71 \\
12.61 & 96.31 \\
18.96 & $\mathbf{3 . 2 8}$ \\
\hline Positive Grid - Negative Source \\
8.94 & 31.14 \\
12.23 & 92.7 \\
18.95 & $\mathbf{3 . 2 9}$ \\
\hline Negative Grid - Positive Source \\
8.72 & $\mathbf{2 5 . 4 5}$ \\
12.62 & 69.49 \\
18.99 & $\mathbf{1 . 2 9}$ \\
\hline Negative Grid - Negative Source \\
8.59 & $\mathbf{2 4 . 4 7}$ \\
12.31 & 66.09 \\
18.99 & $\mathbf{1 . 2 9}$
\end{tabular}
connected branches), we observe some patterns assembled below.

The number of resonant frequencies is generally increasing for the topology cases with more branches. This applies to VS (Table 3) and CS-WT model, however number of resonances in the $\mathrm{Z}(\mathrm{s})$ model stays the same. The newly detected resonances for VS and CS models occur in the proximity of one of the previously detected resonances, therefore they might be considered as the resonances coming from the same respective buses of new branches. The analysis of participation factors in the HRMA methods confirms the consistency in the source of those resonances, ${ }_{595}$ therefore they might be considered as single resonance re-596 gion.

In case of $\mathrm{Z}(\mathrm{s})$ model, the introduction of new branches598 does not introduce the new resonances in the proximity599 of the previous one what implies better accuracy of that600 model in case of multiple branches (and thus multiple Z(s)601 converters) case. On the other hand, the Z(s) model does602 not detect resonances in the low order region as explained603 in the Section 5.3.

Also, for further cases in all models, we observe evident ${ }_{605}$ downward switching of resonant frequencies (Figure 12).606 The switching is caused by higher capacitance (capacitive607 power) for topology cases with more branches connected. 608

By the inspection of participation factors we can clearly609 identify the buses which influence the most particular res-610 onances, as well as, the symmetries in the network. Even611 though the participation factors in some cases have some612 unexpected values, the symmetry and the pattern for their 613 distribution between the branches after new branches con-614 nection is expected and clearly visible (Figures 11 and 13).615
Regarding the stability analysis, for all topology cases the highest group of resonant frequencies (around and 23th or 18th frequency order) is marked as unstable (Table 4). Moreover, for all resonances without exceptions, we observe progressively lower stability margins for the higher topology cases [21]. For the topology Case 3, some resonances becomes marginally stable.

Finally, we observe one, very stiff resonant frequency (or frequencies) which has a source almost completely near the wind turbine inverters (values of PF's mark the source unambiguously). The frequency value of those resonances are very resistant to the influence of capacitance. The introduction of new branches does not shift those frequencies significantly, comparing to the other resonant frequencies. Finally, the stability margin of those resonances is very high, but is also exposed to decrease for the higher topology cases. As mentioned, the source of the resonance is identified near the aggregated wind turbines. In our simulations we do not modify the impedances of aggregated wind turbine, therefore the observations of such a stiff resonance close to the aggregated wind turbines is expected.

\subsection{Comparison of methods}

Table 5 presents the key outputs of the utilized methods. All methods are performed in frequency domain.

Table 5: Comparison of the methods.

\begin{tabular}{lllll} 
& $\begin{array}{l}\text { Series } \\
\text { resonance } \\
\text { detection }\end{array}$ & $\begin{array}{l}\text { Parallel } \\
\text { resonance } \\
\text { detection }\end{array}$ & $\begin{array}{l}\text { Resonance } \\
\text { source de- } \\
\text { tection }\end{array}$ & $\begin{array}{l}\text { Stability } \\
\text { assess- } \\
\text { ment }\end{array}$ \\
\hline \hline $\begin{array}{l}\text { Frequency } \\
\text { Sweep }\end{array}$ & YES & YES & NO & NO \\
HRMA & NO & YES & YES & $\begin{array}{l}\text { YES, } \\
\text { limited }\end{array}$ \\
$\begin{array}{l}\text { Nyquist/ } \\
\text { Bode }\end{array}$ & NO & YES & NO & YES \\
\hline
\end{tabular}

First of all, in almost all cases the HRMA method confirms values of frequencies obtained in FS (Table 1 and Table 2). In some cases it gives even more resonance points. Such differences are observed in case of $\mathrm{Z}(\mathrm{s})$ model, where the FS method is not able to detect low-order resonances. This occur most probably due to the design of the $\mathrm{Z}(\mathrm{s})$ model. Additionally, it can be justified due to the fact that HRMA only considers the dynamic characteristic of the electrical system which is the "denominator" of the transfer function (i.e. the poles are the eigenvalues in HRMA); conversely, FS takes into consideration both the "numerator" and "denominator" (i.e. zeros and poles of the transfer function), leading to pole cancellations or damping. The resonant frequencies obtained from the Bode diagram confirm the values from FS and HRMA; the values are even more consistent for higher frequency orders. These occur likely due to the fact that for high orders the harmonic resonance analysis methods perform much better than for low orders since that is their prime region of analysis.

The series resonance, which we obtain from FS only, is usually considered as less critical in the area of higher 
frequency orders, the parallel resonance therefore is not 654 considered as vital output. In this situation, the method ${ }_{655}$ of Frequency Sweep could be excluded, however due to656 its simplicity and additional information about series res-657 onance could be also useful as an introduction to further ${ }_{658}$ analysis.

The HRMA is definitely crucial due to information ${ }_{660}$ about the sources of resonances (PF's in the Figure $11_{661}$ and Figure 13). The other methods do not give any infor-662 mation about that.

Regarding the stability assessment (Table 4), the anal-664 ysis of Nyquist stability criterion described in the pa-665 per gives clear information about the quality of stability,666 which is easily visible in the Bode diagram. Therefore, the 667 method is considered as very useful in such a study. The 668 stability could also be assessed based on the eigenvalues of network admittance matrix; however, using the eigen-669 values is less intuitive and then the introduction of safety ${ }_{670}$ margin is difficult.

\subsection{Comparison of models}

A comparison of the power converter models applied is674 summarized in this section. Table 6 shows some observa-675 tions regarding the models for analysis.

Table 6: Comparison of the models.

\begin{tabular}{|c|c|c|c|c|c|c|}
\hline & $\begin{array}{l}\text { Resonance } \\
\text { quantity }\end{array}$ & \multicolumn{2}{|c|}{$\begin{array}{l}\text { FS vs. } \\
\text { HRMA }\end{array}$} & \multicolumn{2}{|c|}{$\begin{array}{l}\text { FS\& HRMA } \\
\text { vs. Nyquist }\end{array}$} & $\operatorname{Imp}$ \\
\hline $\begin{array}{l}\text { VS \& } \\
\text { CS-WT }\end{array}$ & $\begin{array}{l}\text { Increases } \\
\text { with } \\
\text { amount } \\
\text { of branches }\end{array}$ & \multicolumn{2}{|c|}{$\begin{array}{l}\text { Complete } \\
\text { match }\end{array}$} & \multicolumn{2}{|c|}{$\mathrm{n} / \mathrm{a}$} & no \\
\hline \multirow[t]{2}{*}{$\mathrm{Z}(\mathrm{s})$} & $\begin{array}{l}\text { Does } \\
\text { not in- } \\
\text { crease with } \\
\text { amount of } \\
\text { branches } \\
\text { (See Sec- } \\
\text { tion } 5.3)\end{array}$ & & $\begin{array}{l}\text { y good } \\
\text { tch, only } \\
\text { missing } \\
\text { quencies } \\
\text { FS case }\end{array}$ & & $\begin{array}{l}\text { good } \\
\text { for } \\
\text { for }\end{array}$ & yes \\
\hline & \multicolumn{2}{|c|}{$\begin{array}{l}\text { Downward shift- } \\
\text { ing }\end{array}$} & \multicolumn{2}{|c|}{$\begin{array}{l}\text { Participation } \\
\text { Factor }\end{array}$} & \multicolumn{2}{|c|}{$\begin{array}{l}\text { "Stiff" } \\
\text { Resonance }\end{array}$} \\
\hline $\begin{array}{l}\text { VS \& } \\
\text { CS-WT }\end{array}$ & \multirow[t]{2}{*}{$\begin{array}{l}\text { Yes, for all } \\
\text { resonances }\end{array}$} & & \multirow{2}{*}{\multicolumn{2}{|c|}{$\begin{array}{l}\text { Similar incl. } \\
\text { dominant bus }\end{array}$}} & \multirow{2}{*}{\multicolumn{2}{|c|}{$\begin{array}{l}\text { Yes, source } \\
\text { near WT } \\
\text { inverters }\end{array}$}} \\
\hline $\mathrm{Z}(\mathrm{s})$ & & & & & & \\
\hline
\end{tabular}

Both well-known approaches of converter modelling by ${ }^{695}$ ideal VS and ideal CS give similar conclusions even though ${ }_{696}^{696}$ the values of resonant frequencies themselves are slightly ${ }^{697}$ different. The sources of resonances indicated by the PF's (Figure 13) and the presence of the stiff resonance are consistent between all three models.

When it comes to the third model $(\mathrm{Z}(\mathrm{s}))$ only, we can ${ }^{702}$ observe some differences, but on the other hand, some pos- ${ }^{702}$ sible advantages over the other two models. The method of frequency sweep for Z(s) yields less resonant frequencies than the other models due to difficulties in the detection of 704 the resonances in the low-order region caused by the $\mathrm{Z}(\mathrm{s})$ model design. Such a blank region does not happen in case ${ }^{705}$ of VS and CS models as they reflect the real dynamics of ${ }^{706}$ the network in the entire frequency range. What is important to highlight, the HRMA method reveal all regions of resonances and is consistent within all three models.

The nonlinear Z(s) model is considered as not fully developed and undoubtedly has more prospective extensions and improvements in contrast to the other two. The converters control can be regulated, therefore the adjustment of the output impedance is possible. In such a way, the level of resonances or the stability margins could be changed and improved to some extent, which is significant advantage in both modelling and operation. Regarding the $\mathrm{Z}(\mathrm{s})$ model, in FS method we observe an interesting phenomenon of resonance below the fundamental frequency (subsynchronous resonance), however, this aspect is not the subject of the paper.

\subsection{Conclusions}

The paper presents the results of impedance analysis of resonance phenomenon in offshore wind power plants, which are decoupled from the main grid due to HVDC transmission. The observations, as well as initial conclusions are provided in the sections above. The final conclusions are summarized in this section.

Three topology cases are taken into consideration for three different models of converters. The view of the harmonic resonance detection in presence of different topologies reveals important facts about the utilized methods and models. It also provide some knowledge about the symmetries in the network. The usefulness of the HRMA method in the context of the topology through the participation factor is very insightful for the harmonic resonance sources detection. Finally, the analysis through the presented methods, makes way for prediction of resonances in case of topology modification, including assessment of resonance origins and stability.

Different methods are studied in order to evaluate their performance in presence of different models and topologies. Such an approach provides more detailed analysis of harmonic resonance. Besides detection of the resonance regions themselves, it shows possible origins of particular resonances and measures the danger for stability. Moreover, some differences between the results of different methods are observed in the performance of different models, therefore the importance of the collective analysis through different methods is emphasised in order to avoid inaccuracies resulting from using an individual method. The deployment of new converter models empower the new resonance mitigation methods, through the control adjustment, possible to investigate as future improvements.

\section{AppendixA. Network data}

Table A.7 presents the values of parameters in the network. 
Table A.7: Data of the WPP network elements converted into the ${ }_{716}$ equivalent voltage level of $150 \mathrm{kV}$.

\begin{tabular}{ccc} 
Component & Symbol & Value at $\mathbf{1 5 0 k V}$ \\
\hline Phase Reactor & $L_{p h}$ & $19.3 \mathrm{mH}$ \\
Tuned C filter & $C_{f}$ & $5.658 \mu \mathrm{F}$ \\
Converter Transformer & $L_{t r 3}$ & $19.338 \mathrm{mH}$ \\
& $R_{c a b 2}$ & $0.056 \Omega$ \\
Cable $150 \mathrm{kV}$ & $L_{c a b 2}$ & $1 \mathrm{mH}$ \\
& $C_{c a b 2}$ & $0.52 \mu \mathrm{F}$ \\
MV/HV Transformer & $L_{t r 2}$ & $38.676 \mathrm{mH}$ \\
& $R_{c a b 1}$ & $0.372 \Omega$ \\
Cable 33kV & $L_{c a b 2}$ & $18.181 \mathrm{mH}$ \\
& $C_{c a b 1}$ & $57.09 \mathrm{nF}$ \\
LV/MV WT Transformer & $L_{t r 1}$ & $51.568 \mathrm{mH}$ \\
& $L_{L C L 1}$ & $1.2 \mathrm{H}$ \\
LCL filter & $L_{L C L 2}$ & $0.641 \mathrm{H}$ \\
& $C_{L C L}$ & $149.1 \mathrm{nF}$
\end{tabular}

\section{AppendixB. Power converters data}

Tables B.8 and B.9 present the values of parameters724 which are implemented to the models described by Equa- ${ }^{-25}$ tions (11) and (15). The values of these parameters ${ }_{727}^{726}$ are obtained from [11], however we rescale the resulting ${ }_{728}$ impedance into the $150 \mathrm{kV}$ equivalent circuit level. Theser29 changes are vital in order to align the impedance to fur $^{-730}$ ther analysis where we combine the converter models with ${ }_{732}^{731}$ the other elements of the network.

Table B 8: Numerical data necessary for calculation of agregated 734 WT converter model (inverter).

\begin{tabular}{ccc} 
Component & Symbol & Value \\
\hline DC Bus voltage & $V_{0}$ & $1500 \mathrm{~V}$ \\
Phase Voltage & $V_{1}$ & $563 \mathrm{~V}$ \\
Phase Current & $I_{1}$ & $236 \mathrm{kA}$ \\
Phase LCL inductance & $L_{1}$ & $25.3 \mu \mathrm{H}$ \\
Current Control $H_{i}(s)$ & $K_{p}$ & $0.44 \cdot 10^{-6}$ \\
& $K_{i}$ & $0.55 \cdot 10^{-3}$ \\
PLL Control $H_{v}(s)$ & $K_{p}$ & 0.239 \\
& $K_{i}$ & 45
\end{tabular}

Table B.9: Numerical data necessary for calculation of HVDC-link $k_{751}$ converter model (rectifier).

\begin{tabular}{ccc} 
Component & Symbol & Value \\
\hline HVDC Link DC voltage & $V_{d c}$ & $300 \mathrm{kV}$ \\
Phase Inductance & $L_{p h}$ & $19.3 \mathrm{mH}$ \\
AC Tuned Filter Capacitance & $C$ & $5.658 \mu \mathrm{F}$ \\
Current Control $H_{i}(s)$ & $K_{p}$ & $0.075 \cdot 10^{-3}$ \\
& $K_{i}$ & 0.094 \\
Voltage Control $H_{v}(s)$ & $K_{p}$ & $11.1 \cdot 10^{-3}$ \\
& $K_{i}$ & 8.388
\end{tabular}

\section{AppendixC. Admittance matrix of HRMA}

The following matrix is the admittance matrix $\mathrm{Y}$ for the HRMA of topology Case 2 of the study case. It has dimension of $11 \times 11$, while the matrices of Case 1 and 3 have the dimension of $7 \times 7$ and $20 \times 20$, respectively.

$\left[\begin{array}{ccccccccccc}y_{11} & -y_{12} & 0 & 0 & 0 & 0 & 0 & 0 & 0 & 0 & 0 \\ -y_{12} & y_{22} & -y_{23} & 0 & 0 & 0 & 0 & 0 & 0 & 0 & 0 \\ 0 & -y_{23} & y_{33} & -y_{34} & 0 & 0 & 0 & -y_{38} & 0 & 0 & 0 \\ 0 & 0 & -y_{34} & y_{44} & -y_{45} & 0 & 0 & 0 & 0 & 0 & 0 \\ 0 & 0 & 0 & -y_{45} & y_{55} & -y_{56} & 0 & 0 & 0 & 0 & 0 \\ 0 & 0 & 0 & 0 & -y_{56} & y_{66} & -y_{67} & 0 & 0 & 0 & 0 \\ 0 & 0 & 0 & 0 & 0 & -y_{67} & y_{77} & 0 & 0 & 0 & 0 \\ 0 & 0 & -y_{38} & 0 & 0 & 0 & 0 & y_{88} & -y_{89} & 0 & 0 \\ 0 & 0 & 0 & 0 & 0 & 0 & 0 & -y_{89} & y_{99} & -y_{9,10} & 0 \\ 0 & 0 & 0 & 0 & 0 & 0 & 0 & 0 & -y_{9,10} & y_{10,10} & -y_{10,11} \\ 0 & 0 & 0 & 0 & 0 & 0 & 0 & 0 & 0 & -y_{10,11} & y_{11,11}\end{array}\right]$

[1] REN21, Renewables 2016 - Global Status Report. Renewable Energy Policy Network for the 21st Century, 2016.

[2] EWEA, The European offshore wind industry - key trends and statistics 2015, European Wind Energy Association, February 2016.

[3] C. Buchhagen, C. Rauscher, A. Menze, J. Jung, BorWin1-First Experiences with harmonic interactions in converter dominated grids, International ETG Congress 2015; Die EnergiewendeBlueprints for the new energy age.

[4] J. C. Das, Power System Harmonics and Passive Filter Designs, IEEE Press Series on Power Engineering, Wiley, 2015.

[5] F. C. De La Rosa, Harmonics and Power Systems, Electric Power Engineering Series, CRC Press, 2006.

[6] G. F. Reed, H. A. Al Hassan, M. J. Korytowski, P. T. Lewis, B. M. Grainger, Comparison of HVAC and HVDC solutions for offshore wind farms with a procedure for system economic evaluation, Energytech, May 2013 IEEE.

[7] J. Arrillaga, N. R. Watson, Power System Harmonics, Wiley InterScience electronic collection, Wiley 2004.

[8] I. J. Pérez-Arriaga, G. C. Verghese, F. C. Schweppe, Selective modal analysis with applications to electric power systems, Part I: Heuristic introduction, IEEE Transactions on Power Apparatus and Systems, no. 9, p. 3117-3125, IEEE 1982.

[9] M. Bradt, B. Badrzadeh, E. Camm, D. Mueller, J. Schoene, T. Siebert, T. Smith, M. Starke, R. Walling, Harmonics and resonance issues in wind power plants, Transmission and Distribution Conference and Exposition (T D), 2012 IEEE PES.

[10] W. Xu, Z. Huang, Y. Cui, H. Wang, Harmonic resonance mode analysis, IEEE Transactions on Power Delivery, 2005, vol. 20, no. 2, p. 1182-1190, April 2005.

[11] H. Liu, J. Sun, Voltage stability and control of offshore wind farms with $A C$ collection and HVDC transmission, IEEE Journal of emerging and selected topics in power electronics, vol. 2, no. 4, p. 1181-1189, IEEE 2014.

[12] J. Sun, Impedance-based stability criterion for grid-connected inverters, IEEE Transactions on Power Electronics, vol. 26, no. 11, p. 3075-3078, IEEE 2011.

[13] R. D. Middlebrook, Input filter considerations in design and application of switching regulators, IAS 1976.

[14] H. Liu, J. Sun, A study of offshore wind HVDC system stability and control Proceedings of 10th International Workshop on Large-Scale Integration of Wind Power into Power Systems as well as on Transmission Networks for Offshore Wind Power Plans, 2011

[15] R. Bellman, Introduction to matrix analysis, University of Southern California, vol. 960, SIAM 1970.

[16] G. C. Verghese, I. J. Perez-Arriaga, F. C. Schweppe, Selective Modal Analysis With Applications to Electric Power Systems, Part II: The Dynamic Stability Problem, IEEE Transactions on Power Apparatus and Systems, vol. PAS-101, no. 9, p. 31263134, September 1982.

[17] J. Sun, Small-signal methods for AC distributed power systemsa review, IEEE Transactions on Power Electronics, vol. 24, no. 11, p. 2545-2554, IEEE 2009. 
[18] Z. Bing, K. Karimi, J. Sun, Input impedance modeling and analysis of line-commutated rectifiers, IEEE Transactions on Power Electronics, vol. 24, no. 10, p. 2338-2346, IEEE 2009.

[19] M. Cespedes, J. Sun, Impedance modeling and analysis of grid-connected voltage-source converters, IEEE Transactions on Power Electronics, vol. 29, no. 3, p. 1254-1261, IEEE 2014.

[20] E. Larsen, G. Drobnjak, H. Elahi, Standardization of VSC$H V d c$ interface with offshore wind generation, Proc. 11th Int. Workshop Large-Scale Integr. Wind Power Power Syst., p. 169$174,2012$.

[21] I. Sowa, Impedance analysis of harmonic resonance in HVDC connected Wind Power Plants Master Thesis, Escola Tècnica Superior d'Enginyeria Industrial de Barcelona, UPC 2016. 